\title{
3D Shape Analysis of Intracranial Aneurysms Using the Writhe Number as a Discriminant for Rupture
}

\author{
Alexandra Lauric, ${ }^{1}$ Eric L. Miller, ${ }^{2}$ Merih I. Baharoglu, ${ }^{3}$ and Adel M. Malek ${ }^{3}$ \\ ${ }^{1}$ Department of Computer Science, Tufts University, 161 College Ave, Medford, MA 02155, USA; ${ }^{2}$ Department of Electrical \\ and Computer Engineering, Tufts University, Medford, MA, USA; and ${ }^{3}$ Department of Neurosurgery, Tufts Medical Center, \\ Boston, MA, USA
}

(Received 17 September 2010; accepted 23 December 2010)

Associate Editor Joan Greve oversaw the review of this article.

\begin{abstract}
Intracranial aneurysms are polymorphic focal arterial dilations, which harbor a variable risk of rupture leading to high morbidity and mortality. Increased detection of incidental aneurysms by non-invasive imaging has created a need for rupture risk stratification tools, in addition to simple aneurysm size, to guide optimal treatment strategy. To this end, shape analysis has emerged as a possible differentiator of rupture likelihood. A novel set of morphological parameters based on the writhe number are introduced here to describe aneurysms and discriminate rupture status. Classification in 117 saccular aneurysms (52 ruptured and 65 unruptured) is based on statistical analysis of writhe number distribution on the aneurysm surface. Aneurysms are analyzed both in isolation and including a portion of their parent vessel. Sidewall and bifurcation aneurysm subtypes were found to be best described by disjoint sets of shape parameters, yielding a morphological dichotomy between the two aneurysm classes. Writhe number analysis results in $86.7 \%$ accuracy on sidewall (SW) aneurysms and $71.2 \%$ accuracy on bifurcation (BF) aneurysms. This represents a $12 \%$ accuracy increase for both subtypes compared to the performance of seven established $2 \mathrm{D}$ and $3 \mathrm{D}$ indexes. The results support the utility of writhe number aneurysm shape analysis, with potential clinical value in rupture risk stratification.
\end{abstract}

Keywords-Intracranial aneurysms, Morphological characterization, Histogram statistics, Shape analysis, Writhe number.

\section{INTRODUCTION}

Recent advances in imaging technologies and the wide availability of computed tomography (CT) and magnetic resonance (MR) imaging, have led to

Address correspondence to Alexandra Lauric, Department of Computer Science, Tufts University, 161 College Ave, Medford, MA 02155, USA.Electronic mail: alauri02@cs.tufts.edu, alex.lauric@ gmail.com increased detection of incidental, asymptomatic, unruptured intracranial aneurysms (UIA) during routine evaluation of headache, dizziness, or trauma. ${ }^{24}$ Rupture of these lesions may lead to subarachnoid hemorrhage (SAH), a severe condition associated with high mortality $(10-20 \%)$ and morbidity $(25-50 \%) .^{21}$ While the recommendation is for all symptomatic aneurysms to be treated, the management of asymptomatic UIA remains controversial. ${ }^{21,23}$ Recent studies estimate a significantly lower annual rupture risk of $0.1-0.2 \%$ for UIA, ${ }^{22}$ in contrast to earlier reports of $1-2 \%$ annual rupture rates. ${ }^{21}$ Since preventive treatment carries risks of complications which increase with age, the decision to intervene and treat UIA needs to be balanced against the risk of rupture.

Predicting rupture in UIA has focused mainly on aneurysm size and other two-dimensional indexes such as aspect ratio (AR), height-width (HW) ratio, and the bottleneck factor (BN)..$^{9,12,20}$ However, many small aneurysms rupture, whereas some large aneurysms never do. ${ }^{16}$ Cerebral aneurysms present in many shapes and sizes. Like size, shape is likely to influence the rupture risk. ${ }^{4,16}$ Advances in imaging modalities such as 3D rotational angiography (3D-RA), CT, and $\mathrm{MR}$, makes it possible to analyze the complex shape of aneurysms in a 3D environment. Some of the first morphological indexes, such as non-sphericity, ellipticity, and undulation were proposed by Raghavan et al. ${ }^{16}$ and studied further in subsequent studies. ${ }^{4,9}$ Rohde et al. ${ }^{17}$ applied Fourier analysis to capture the shape irregularities of intracranial aneurysms. Their Fourier analysis was performed on 2D data sets. They evaluated manually segmented contours of aneurysms defined on projection planes subjectively chosen to best show the relationship between aneurysms neck and the parent vessel. Finally, Millan et al. ${ }^{13}$ introduced 3D geometrical and Zernike moment invariants to 


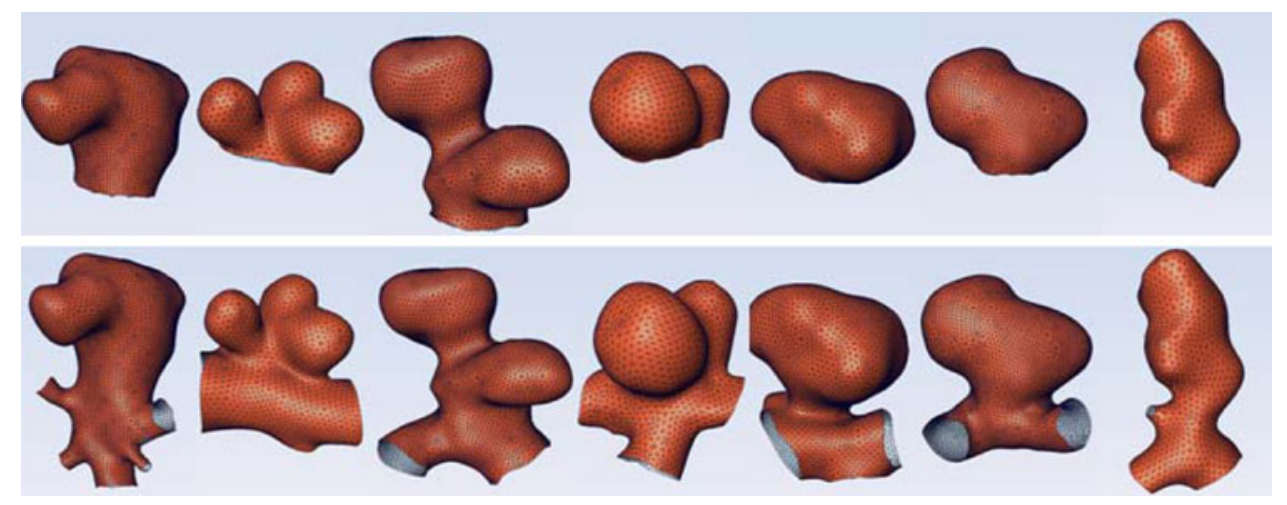

FIGURE 1. Aneurysm surfaces are analyzed both completely isolated from parent vessels and taking into account a portion of their adjacent vessels. They are represented as triangular meshes. Both bifurcation and sidewall aneurysms are considered. First row shows isolated aneurysms. Second row shows the aneurysm attached to its corresponding parent vessels.

describe the 3D shape of the aneurysms in an automatic fashion.

The purpose of the current work is to explore the potential of geometrical characterization of cerebral aneurysms in differentiating between ruptured and unruptured aneurysms. To this end, we introduce a series of original shape descriptors based on the writhe number. ${ }^{6}$ Similar to recent morphological studies, ${ }^{9,12,13,16,17}$ the rupture status prediction is performed on a data set of aneurysms, which were labeled as being ruptured or not at the time of detection by the treating neurosurgeons. In the proposed methodology, aneurysms are isolated from the segmented vasculature represented as a 3D triangular mesh. They are analyzed both as completely isolated lesions and also by inclusion of their adjacent parent vessels (Fig. 1). Writhe numbers are computed for vertices along the surface. Known in curve theory since its introduction by Fuller, ${ }^{6}$ the writhe number was recently extended to 3D surfaces. ${ }^{11}$

There,$^{11}$ the writhe number of surfaces was used as a binary parameter to distinguish between healthy vessels and possible aneurysmal regions. Healthy vessels were modeled as tubular structures which have zero writhe numbers. Consequently, those portions of the vessels where the writhe number was non-zero were reported as possible aneurysms. Here, we proposed a different use of the writhe number and regard the writhe number as a function defined on the whole aneurysm surface. The range of writhe values along the surface is highly dependent on the aneurysm geometry. The resulting writhe number distribution is analyzed through the use of histogram statistics such as central moments, cumulants, and entropy. These measures are used as classification attributes in rupture status discrimination in a dataset of cerebral aneurysms.
The morphological analysis was performed on a database of 117 saccular aneurysms (52 ruptured and 65 unruptured) derived from 3D-RA imaging studies. The aneurysms were further labeled as SW (58 aneurysms) or BF (59 aneurysms) according to their location with respect to the parent vessels. It was found that SW and BF aneurysms were best described by disjoint sets of shape parameters and we report a morphological dichotomy between these two aneurysm subtypes. Incorporating parent vessel information increased the ability to discriminate rupture status for both SW and BF aneurysms. The rupture status classification results were compared with the performance of seven established 2D (size, AR, HW, and $\mathrm{BN}$ ) and 3D (ellipticity, undulation, and non-sphericity) indexes.

The article is structured as follows: the writhe number theory is presented in "The Writhe Number" section. "Materials and Methods" section describes our materials and methodology. Results and robustness analysis are detailed in "Results" section. Discussion, conclusions, and directions for future work are presented in "Discussion" section.

\section{THE WRITHE NUMBER}

\section{The Writhe of Surfaces}

The writhe number was introduced by Fuller ${ }^{6}$ and it is used in curve theory to measure the extent to which a curve twists and coils around itself. In biomedical engineering, the $2 \mathrm{D}$ writhe number is used to study the shape and topology of $\mathrm{DNA}^{18}$ or to characterize the shape of curves on 3D surfaces, such as the curves of sulci and gyri on the cortical surface. ${ }^{10}$

Let $C$ be a closed non-self-intersecting smooth curve $C=r(s):[0, L] \rightarrow \Re^{3}$, parameterized by arclength $s$. 
The writhe number $W_{r}$ of a curve is calculated from the tangents to the curve as the double line integral:

$$
W_{r}(C)=\frac{1}{4 \pi} \int_{0}^{L} \int_{0}^{L} \frac{\left[\mathbf{t}_{\mathbf{s}_{1}}, r\left(s_{2}\right)-r\left(s_{1}\right), \mathbf{t}_{\mathbf{s}_{2}}\right]}{\left\|r\left(s_{2}\right)-r\left(s_{1}\right)\right\|^{3}} d s_{1} d s_{2},
$$

where $s_{1}, s_{2}$ are arclengths and $\mathbf{t}_{\mathbf{s}}=r^{\prime}(s)$ is the tangent vector. The relation $[\mathbf{a}, \mathbf{b}, \mathbf{c}]$ is the triple scalar product of vectors $\mathbf{a}, \mathbf{b}$, and $\mathbf{c}$. The triple scalar product is defined as $[\mathbf{a}, \mathbf{b}, \mathbf{c}]=\mathbf{a} \cdot(\mathbf{b} \times \mathbf{c})$, where $\mathbf{a} \cdot \mathbf{b}$ denotes a dot product and $\mathbf{a} \times \mathbf{b}$ denotes a cross product.

A generalization of Eq. (1), from curves to surfaces, was proposed in recent research ${ }^{11}$ and applied to detecting aneurysms from cerebral vascular trees. Motivated by the form of the integrand in Eq. (1), for two points $\mathbf{p}$ and $\mathbf{p}^{\prime}$ on surface $S$, we define a relationship $w$ between them as

$$
w\left(\mathbf{p}, \mathbf{p}^{\prime}\right)=\frac{\left[\mathbf{n}_{\mathbf{p}}, \mathbf{p}^{\prime}-\mathbf{p}, \mathbf{n}_{\mathbf{p}^{\prime}}\right]}{\left\|\mathbf{n}_{\mathbf{p}}\right\| \cdot\left\|\mathbf{p}^{\prime}-\mathbf{p}\right\| \cdot\left\|\mathbf{n}_{\mathbf{p}^{\prime}}\right\|},
$$

where $\mathbf{n}_{\mathbf{p}}$ is the surface normal at point $\mathbf{p},\|\cdot\|$ is the norm of a vector. The writhe number of a point $\mathbf{p}$, given a surface $S$ with $\mathbf{p} \in S$, is defined as

$$
W(\mathbf{p}, S)=\int_{\mathbf{p}^{\prime} \in S} w\left(\mathbf{p}, \mathbf{p}^{\prime}\right) d S,
$$

which by discretization becomes

$$
W(\mathbf{p}, S)=\sum_{\mathbf{p}^{\prime} \in S} w\left(\mathbf{p}, \mathbf{p}^{\prime}\right) \Delta \mathbf{p}^{\prime},
$$

In this work, $S$ is a triangular mesh and $\Delta \mathbf{p}^{\prime}$ is the area associated with vertex $\mathbf{p}^{\prime}$. The writhe number depends on both the shape and the size of the surface and has units of $\left[\mathrm{Length}^{2}\right]$, in our case $\left[\mathrm{mm}^{2}\right]$. A scale analysis of the writhe number is provided in "Sensitivity to Scaling" section. The differential surface area, $\Delta \mathbf{p}^{\prime}$, is computed as one-third of the sum area of all triangles defined by $\mathbf{p}^{\prime}$.

\section{A Geometric Interpretation of the Writhe Number}

Using the pseudoscalar quality of the writhe number, it was previously proved ${ }^{11}$ that the writhe number of a point on a cylinder is zero. Similarly, it can be proved that the writhe of other symmetrical shapes, such as spheres, is also zero.

More generally, given a surface $S$ and a point $\mathbf{p} \in S$ on the surface, $W(\mathbf{p}, S)=0$ if $S$ displays mirror symmetry about a mirror plane which passes through $\mathbf{p}$ and contains the normal at $\mathbf{p}$. Mirror symmetry occurs when two halves of a whole are each other's mirror images and guarantees that for every point $\mathbf{p}_{\mathbf{1}} \in S$ there exists a second point $\mathbf{p}_{2} \in S$, such that the Euclidean distance $\left|\mathbf{p}_{1}-\mathbf{p}\right|=\left|\mathbf{p}_{\mathbf{2}}-\mathbf{p}\right|$, with $\mathbf{p}_{1}$ and $\mathbf{p}_{\mathbf{2}}$ located on different sides of the mirror plane. ${ }^{11}$ The normals at $\mathbf{p}_{\mathbf{1}}$ and $\mathbf{p}_{\mathbf{2}}$ cancel each other's contribution to the writhe number summation.

This notion of symmetry captured by the writhe number was previously used for aneurysm detection in order to distinguish between normal vessels (modeled locally as cylinders) and aneurysmal regions. ${ }^{11}$ In this current work, writhe number distribution along an aneurysmal surface is used to capture surface asymmetries and is analyzed through the use of histogram statistics.

\section{A Physical Interpretation of the Writhe Number}

In addition to the geometric interpretation of the writhe number, we also introduce a mechanical interpretation of the writhe number by analogy with the mechanical torque.

The torque, also called the moment of force, describes the rotational effect of an external force applied on a rigid body $S$. The torque $\tau$ about a point $O$ acting on $S$ under the influence of an external force $\mathbf{F}$ is defined as $\tau=\mathbf{r} \times \mathbf{F}$. Vector $\mathbf{r}$ is the distance vector between the origin of the torque, point $O$, and the point where $\mathbf{F}$ is applied. ${ }^{1}$ Similarly, the torque acting on $S$ under the influence of an external force $\mathbf{F}$ about an arbitrary axis defined by the unit vector $\mathbf{n}$ is defined as $\tau=\mathbf{n} \cdot(\mathbf{r} \times \mathbf{F}) .{ }^{1}$ In other words, $\tau=[\mathbf{n}, \mathbf{r}, \mathbf{F}]$ is the triple scalar product of vectors $\mathbf{n}, \mathbf{r}$, and $\mathbf{F}$.

For the purposes of this work, $S$ is the surface of an aneurysm described as a collection of $3 \mathrm{D}$ vertices, $\mathbf{p}_{\mathbf{i}} \in S$, with normals $\mathbf{n}_{\mathbf{i}}$. Unit forces act at the vertices of $S$ in the direction of their surface normals, creating a discretized force field surrounding the aneurysm. When computing the writhe of a particular point $\mathbf{p}$, the normal $\mathbf{n}$ at $\mathbf{p}$ is considered the rotation axis of the torque.

To better reflect the relation between the writhe of a point and the torque at that point, the writhe of a point p can be expressed as a function of the torque about the normal at point $\mathbf{p}: W(\mathbf{p}, S)=\sum_{\mathbf{p}_{\mathbf{i}} \in S}^{N} \frac{\tau_{i}}{\left\|\mathbf{p}_{\mathbf{i}}-\mathbf{p}\right\|} \Delta p_{i}$. In effect, we are computing a normalized torque along the normals of all the points on the surface of the aneurysm. Using the analogy with the torque, it can be concluded that the writhe number of a point $\mathbf{p}$ is, in a sense, proportional to the tendency of the normals to impart to the aneurysm a motion of rotation about a fixed axis $\mathbf{n}$ along the normal at point $\mathbf{p}$.

The torque is a measure of the "twisting force" acting on an object. ${ }^{14}$ In our case, this twisting force is measured along the whole surface of an object. As previously described, symmetrical objects have zero writhe numbers and zero net twisting forces at all 
surface points. Throughout this work then, the writhe number can be viewed more or less as a measure of how close the aneurysm is to equilibrium at each point on its surface. In other words, the writhe number measures how much "twisting force" acts at each point on the surface of the aneurysm. Intuitively, the results provided in this work indicate that the more spread and the stronger the "twisting force" is on the surface, the greater the likelihood for rupture is. The usefulness of the writhe number is discussed in "Rupture Status Classification" section.

\section{MATERIALS AND METHODS}

\section{Data Description}

During a 4-year period (2001-2005), patient-derived 3D-RA data were collected for patients with intracranial aneurysms treated at Tufts Medical Center, Department of Neurosurgery (Boston, MA). Data were acquired using a biplane flat-detector digital subtraction angiography system (Axiom Artis, Siemens Medical Solutions, Malvern, PA). 3D-RA is a technique employed to visualize blood vessels in a bony or dense soft tissue environment. In the current study, the size of each 3D-RA data volume is $128 \times 128 \times 128$ voxels with $0.48 \mathrm{~mm}$ isotropic spacing. Approval for the collection and review of data was obtained from the Institutional Review Board at Tufts Medical Center.

Similar to other studies, ${ }^{4}$ only cases in which the rotational angiographic images were of sufficient quality for accurate segmentation and reconstruction were included in the study. A number of 106 consecutive patients, resulting in 117 distinct cerebral aneurysms, met these criteria. Rupture status was recorded at the time of presentation by the treating neurosurgeons and the 117 aneurysms were classified as ruptured (52 aneurysms) and unruptured (65 aneurysms). The aneurysms were further classified as SW (dilation of the artery in one direction perpendicular to the vessel axis58 aneurysms) and BF (dilation at the BF of arteries- 59 aneurysms). Fusiform aneurysms are not considered in this study given how different their pathology and morphology are from that of saccular aneurysms. ${ }^{3}$

The distribution of the aneurysms according to their location, rupture status, and relation with parent vessel is shown in Table 1 together with the mean values of their largest diameter size derived from manual measurements in $3 \mathrm{D}$ space. The average maximal size of the aneurysms in the database is $6.8 \pm 3.1 \mathrm{~mm}$.

\section{Existing Size and Shape Indexes}

The shape index proposed in this study is compared with the following existing $2 \mathrm{D}$ and $3 \mathrm{D}$ indexes which are described in this section.

2D indexes ${ }^{9,16,20}$ : The largest diameter of the aneurysm is a commonly used index to predict rupture (Fig. 2a). The AR (aneurysm height/neck width) is another potentially useful size index used to estimate the risk of rupture, especially for small aneurysms which might be missed by the largest diameter measure (Fig. 2b). Other commonly used 2D size descriptors which were found to be associated with rupture risk are the HW index and the BN index. HW is defined as the height of the aneurysm divided by its largest diameter (Fig. 2c), while BN is defined as the width of the aneurysm divided by its neck diameter (Fig. 2d). For this work, the size indexes described above were measured manually by an experienced clinical operator. The measurements were performed from the 3D model of each aneurysm, using a commercial 3D visualization and modeling system (Amira, Mercury Systems, Chelmsford, MA).

3D indexes ${ }^{4,16}$ : The ellipticity index $(\mathrm{EI})$ provides a measure of how close the shape of the aneurysm is to an ellipsoid. The EI is defined as $E I=1-(18 \pi)^{\frac{1}{3}} \frac{V_{C H}^{\frac{2}{3}}}{S_{C H}}$, where $V_{C H}$ is the volume of the aneurysm convex hull

TABLE 1. Details about aneurysm distribution based on location, relation with the parent vessel (BF vs. SW) and rupture status.

\begin{tabular}{|c|c|c|c|c|c|c|}
\hline & \multirow[b]{2}{*}{$N$} & \multirow[b]{2}{*}{ Size $(\mathrm{mm})$} & \multicolumn{2}{|c|}{ SW } & \multicolumn{2}{|c|}{$\mathrm{BF}$} \\
\hline & & & Unruptured & Ruptured & Unruptured & Ruptured \\
\hline Posterior communicating (PComA) & 31 & $8.4 \pm 3.8$ & 13 & 11 & 3 & 4 \\
\hline Anterior communicating (AComA) & 22 & $5.4 \pm 2.0$ & & & 3 & 19 \\
\hline Internal carotid (ICA) & 18 & $7.1 \pm 3.6$ & 14 & 4 & & \\
\hline Middle cerebral (MCA) & 17 & $5.9 \pm 1.9$ & & & 16 & 1 \\
\hline Ophthalmic artery (OpthA) & 8 & $7.5 \pm 3.7$ & 5 & 3 & & \\
\hline Internal carotid artery bifurcation (ICAB) & 6 & $5.8 \pm 2.3$ & & & 6 & \\
\hline Basilar & 6 & $7.4 \pm 2.4$ & & & 2 & 4 \\
\hline Anterior choroidal artery $(\mathrm{AChA})$ & 5 & $5.2 \pm 2.0$ & 2 & 3 & & \\
\hline Vertebral (VA) & 3 & $5.9 \pm 1.0$ & 1 & 2 & & \\
\hline Posterior inferior cerebellar artery (PICA) & 1 & 8.1 & & & & 1 \\
\hline
\end{tabular}


(a)

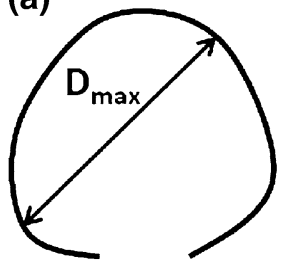

(b)

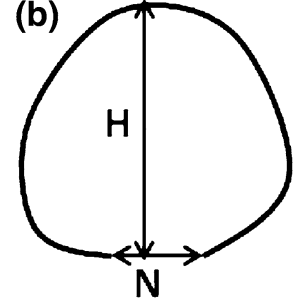

(c)

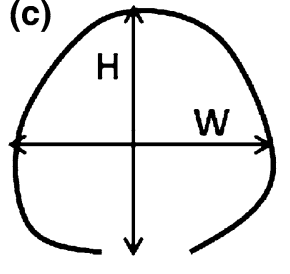

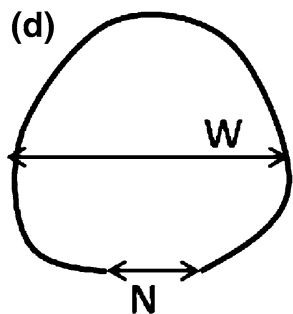

FIGURE 2. Existing 2D size indexes. (a) Largest diameter size. (b) Aspect ratio is defined as the height $H$ of the aneurysm divided by its neck diameter $N$. (c) Height-width index is defined as the height $H$ of the aneurysm divided by its width $W$. (d) The bottleneck factor is defined as the dome width $W$ divided by its neck diameter $N$.

and $S_{C H}$ is the surface of the aneurysm convex hull. The EI varies from 0 to 1 , being 0 for a sphere and increasing with ellipticity. The undulation index (UI) describes how irregular is the surface of the aneurysm. The UI is defined as $U I=1-\frac{V}{V_{C H}}$, where $V_{C H}$ is the volume of the aneurysm convex hull and $V$ is the aneurysm volume. The UI varies from 0 to 1 , being 0 for completely convex aneurysm and increasing with the presence of surface irregularities and lobulations. The non-sphericity index (NSI) measures how closely the shape of the aneurysm resembles a sphere. The NSI is defined as $N S I=1-(18 \pi)^{\frac{1}{3}} \frac{V^{\frac{2}{3}}}{S}$, where $V$ is the aneurysm volume and $S$ is the aneurysm surface. The NSI varies from 0 to 1 , being 0 for a hemisphere and increasing with deviation from a spherical shape due to ellipticity or undulations in the surface. ${ }^{16}$ The EI, UI, NSI, and curvature indexes are some of the first 3D shape descriptors to be associated with aneurysm rupture risk and to be included in relevant clinical research studies. ${ }^{4,9,16}$ For this work, the shape indexes described above were computed automatically from 3D aneurysm models (AMs).

\section{Method}

The following methodology is proposed to differentiate rupture status in cerebral aneurysms. The cerebral vasculature is segmented from the 3D-RA volume. For each aneurysm two separate $3 \mathrm{D}$ models are created, one of the completely isolated aneurysm and one of the aneurysm with some portion of the adjacent vessels included. Models are represented as triangular meshes. Writhe numbers are computed on the surface of the models. Kernel density methods are applied to the histogram of writhe numbers distributions and statistics such as central moments, cumulants, and entropy are computed for the estimated density function. These descriptors are used as features in the classification of rupture status in cerebral aneurysms. Details about each step are presented below.

\section{Segmentation}

Segmentation is performed using a combination of thresholding and region-growing techniques, ${ }^{15}$ applied with a commercial 3D visualization and modeling system (Amira, Mercury Systems, Chelmsford, MA) (Fig. 3b). The surface of the segmented vasculature is described as a triangular mesh (Fig. 3c).

\section{Isolation of Aneurysms}

To allow for morphologic analysis, the aneurysm geometry is manually separated from the segmented vasculature. Each aneurysm is isolated from surrounding vessels in two steps. First, the aneurysm is cut in such a way that its neck and a portion of the adjacent vessels are included in the model. The cut planes are empirically selected, the emphasis being on capturing the whole attachment area (i.e., the region where the aneurysm neck attaches to the parent vessels). Each parent vessel is cut at a distance approximately equal to its diameter, ${ }^{13}$ as measured at the aneurysm neck. Second, the aneurysm sac is completely isolated from all surrounding vessels. The cut plane is selected at the location where the aneurysmal sac pouched outward from the parent vessel. ${ }^{4}$ The two resulting models are represented as triangular meshes as shown in Fig. 3 and the writhe number is computed for each vertex on the aneurysm surface according to Eq. (4).

\section{Histogram Smoothing}

The writhe number values along the surface of each aneurysm are represented in a frequency histogram. The value of each bin is divided by the total number of samples, and the area under the counting bins adds to one. The histogram is a non-smooth estimator of the underlying density function showing discontinuities at its ends and at bins with zero value. To avoid these shortcomings, histogram smoothing is performed using Nadaraya-Watson estimator with Gaussian 
(a)

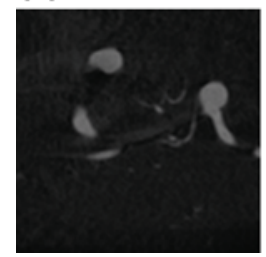

(b)

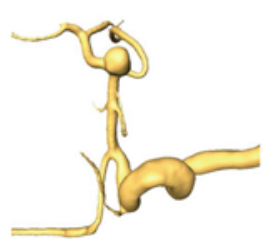

(c)

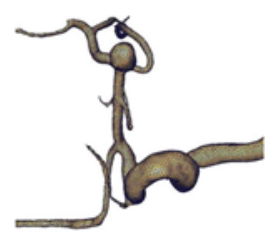

(d)

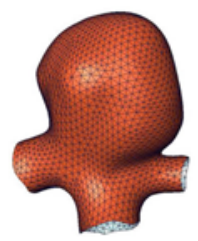

(e)

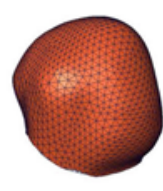

FIGURE 3. Segmentation and isolation of aneurysms. (a) Original 3D-RA data. (b) Cerebral vessels are segmented from surrounding tissue. (c) Segmented vessels are represented as a triangular mesh. (d) The aneurysm is separated from the vasculature and part of the adjacent parent vessels is included. (e) The aneurysm dome is completely separated from its parent vessels.

kernels ${ }^{7}$ whose bandwidth is selected using the method of Bowman et al. ${ }^{2}$

\section{Histogram Statistics}

Statistics such as central moments, cumulants, and entropy are applied to the smoothed histogram to describe and analyze writhe number distributions.

The central moments of a probability density function $p(x)$ are defined as $\mu_{i}=\int_{-\infty}^{\infty}(x-c)^{i} p(x) d x$, where $c$ is the mean of the random variables $x$. The first central moment is zero. The second central moment, $\mu_{2}$, is the variance and describes the distribution width within the values $x_{i}$. The third central moment, $\mu_{3}$, is the skew and characterizes the asymmetry of the shape around the mean. The fourth central moment, $\mu_{4}$, is the kurtosis and measures the sharpness of the distribution.

The first five cumulants as functions of the central moments are: $k_{1}=c, k_{2}=\mu_{2}, k_{3}=\mu_{3}, k_{4}=\mu_{4}-3 \mu_{2}^{2}$ and $k_{5}=\mu_{5}-10 \mu_{3} \mu_{2}$. The fourth order cumulant gives a measure of the non-Gaussianity of the variable $x$. Distributions with sharp peeks and heavy tails have positive $k_{4}$, whereas distributions with flatter shapes have negative $k_{4}$.

The entropy of a continuous random variable $x$, with density $p(x)$, is a measure of the uncertainty associated with that variable and it is defined as $h(x)=-\int_{x} p(x) \log p(x) d x$. The entropy does not depend on the values of $x$ but only on the probabilities that $x$ will occur.

Using high order central moments and cumulants can result in a very large number of features. To control the classification, the number of features was restricted and each model is described by 10 attributes related to the writhe number distribution: the entropy, central moments 2-8 and, cumulants 4 and 5 (cumulants $1-3$ have the same formula as the corresponding moments). Consequently, each natural aneurysm is associated with 20 attributes, 10 describing the aneurysm sac and 10 describing the aneurysm with portion of the parent vessels attached.

\section{Classification}

The classification problem solved here involves two classes (ruptured vs. unruptured) and 117 samples (the aneurysms), described by 20 features each. The method used is logistic regression with 10 -fold cross-validation. The classification is repeated 10 times with 10 different random seeds. This results in 100 different splittings and the average performance is reported.

Classification is first performed on all 117 samples, then separately on $58 \mathrm{SW}$ and $59 \mathrm{BF}$ aneurysms. In each case, first the aneurysm sac attributes are considered, then only the attributes from aneurysm with parent vessels attached are considered, and finally the total of 20 attributes are considered. The results of these nine different scenarios are described in "Rupture Status Classification" section. To the best of our knowledge, this is the first study to perform morphological analysis and compare prediction results on both aneurysm sacs models and parent vessels models.

The best ratio between the number of features and the number of training samples is a controversial issue. A long standing statistical rule-of-thumb suggests that 10 samples per feature are a minimum requirement for regression in order to avoid overfitting. ${ }^{8}$ To reduce the dimensionality of the feature space, sequential backward selection was performed on the number of attributes considered for each of the nine classification scenarios. Details about feature selection for each classification case are provided in "Rupture Status Classification" section.

\section{RESULTS}

\section{Rupture Status Classification}

In this study, the rupture status classification results are compared with those of seven established 2D and $3 \mathrm{D}$ indexes. Table 2 shows the discrimination accuracy of these indexes when used on the whole set of 117 aneurysms (SW $+\mathrm{BF})$ and on subsets of SW and BF aneurysms, respectively. All indexes perform much 
TABLE 2. Accuracy of rupture prediction when aspect ratio (AR), largest diameter size, height-width and aneurysm angle are used.

\begin{tabular}{llll}
\hline Type & $N$ & Features & Accuracy \% \\
\hline SW + BF & 117 & Size & 55.9 \\
SW + BF & 117 & AR & 63.6 \\
SW + BF & 117 & HW & 64.7 \\
SW + BF & 117 & BN & 60.7 \\
SW + BF & 117 & El & 62.5 \\
SW + BF & 117 & UI & 62.3 \\
SW + BF & 117 & NSI & 66.9 \\
SW + BF & 117 & HW, NSI & 68.3 \\
SW & 58 & Size & 70.6 \\
SW & 58 & AR & 69.2 \\
SW & 58 & HW & 67.2 \\
SW & 58 & BN & 67.9 \\
SW & 58 & El & 61.6 \\
SW & 58 & UI & 68.6 \\
SW & 58 & NSI & 77.1 \\
SW & 58 & Size, NSI & 77.1 \\
BF & 59 & Size & 47.7 \\
BF & 59 & AR & 62.1 \\
BF & 59 & HW & 64.0 \\
BF & 59 & BN & 55.0 \\
BF & 59 & El & 62.7 \\
BF & 59 & UI & 62.6 \\
BF & 59 & NSI & 60.2 \\
BF & 59 & AR, HW, UI & 64.2 \\
\hline Th & & & \\
\hline & & &
\end{tabular}

The prediction is performed on three aneurysms sets: (1) 117 sidewall and bifurcation (SW + BF) aneurysms, (2) 58 sidewall (SW) aneurysms, and (3) 59 bifurcation (BF) aneurysms.

better on SW than on BF aneurysms. This is consistent with recent research findings in our lab utilizing conventional aneurysm size and first-order shape analysis that most of the currently used shape and size indexes perform better on SW vs. BF aneurysms. When each index is considered independently in univariate analysis, the rupture in SW aneurysms is best captured by NSI, size, and AR indexes (accuracy 77.1, 70.6, and 69.3, respectively). Using similar analysis, the rupture in BF aneurysms is best described by EI, UI, and AR indexes (accuracy 62.7, 62.6, and 62.1, respectively). It is interesting to note that when the whole set of indexes is used to predict rupture status using multivariate analysis, the final results are not significantly improved compared to the best univariate results, for both SW and BF subsets. The set of indexes identified as being statistically significant for multivariate analysis are also shown in Table 2. They are size and NSI for SW aneurysms, and AR, HW, and UI for BF aneurysms. The results suggest there is some overlap between indexes, with a number of indexes describing the same size or shape quality.

Table 3 summarizes the central results of the study. Classification is performed on the three subsets: 117
$\mathrm{SW}+\mathrm{BF}, 58 \mathrm{SW}$, and $59 \mathrm{BF}$ aneurysms. For each subset, rupture status is predicted by considering first only AM features, second considering only parent vessel model (PVM) features, and third considering both AM and PVM features. These are the features previously detailed in "Method" section. The sets of features taken into account for each particular classification case are marked with an $\mathrm{X}$ in the corresponding columns of the table. We distinguish between the features which are taken into consideration, and those which are ultimately used for classification. More specifically, sequential backward selection is applied on the features taken into consideration to reduce the set to the five most significant features, which are the features used for classification. Using five features guarantees a samples-to-features ratio greater than 10 . In turns, this reduces the risk that multivariate classification may overfit the data.

Best classification results are obtained when the analysis is performed separately on $\mathrm{SW}$ and $\mathrm{BF}$ aneurysms, respectively. The accuracy obtained using morphological analysis based on writhe number is of $86.7 \%$ for SW aneurysms and of $71.2 \%$ for BF aneurysms. Adding parent vessel information increased the classification accuracy for SW aneurysms and proved essential for BF aneurysms.

In terms of features, the entropy is part of all best features sets and it seems to be a significant quantity for both aneurysm subtypes. This suggests that high degrees of randomness within writhe number values (regions of high twisting forces alternating with regions of low twisting forces) are associates with rupture. Indeed, correctly classified ruptured aneurysms are consistently characterized by a higher mean entropy of the writhe number compared to unruptured aneurysms. Also, all best feature sets contain at least two of the variance, skewness and kurtosis central moments.

The differences between ruptured and unruptured aneurysms are apparent in Figs. 4 and 5 which show analysis results on SW and BF aneurysms, respectively. In the figures, we show the writhe number values along the surface of ruptured and unruptured aneurysms and their corresponding histograms. For SW aneurysms the results are shown on aneurysm dome models and for BF aneurysms the results are illustrated on aneurysms with adjacent parent vessels attached. Regions of high writhe numbers (high twisting forces) on the surface of the aneurysms are associated with rupture status. It can be seen from the histograms that unruptured aneurysms have small skewness and small kurtosis (sharp distribution), while ruptured aneurysms tend to have high positive skewness and high kurtosis (flat distribution). 
TABLE 3. Accuracy of rupture prediction when writhe number statistics are used.

\begin{tabular}{|c|c|c|c|c|c|}
\hline Type & $\mathrm{AM}$ & PVM & Best features AM & Best features PVM & Accuracy \% \\
\hline $\mathrm{SW}+\mathrm{BF}$ & $x$ & & $\mu_{2}, \mu_{3}, \mu_{4}, \mu_{5}, h$ & & 67.9 \\
\hline $\mathrm{SW}+\mathrm{BF}$ & & $x$ & & $\mu_{2}, \mu_{7}, \mu_{8}, k_{4}, h$ & 68.3 \\
\hline $\mathrm{SW}+\mathrm{BF}$ & $x$ & $X$ & $\mu_{2}, \mu_{3} \mu_{5}$ & $\mu_{8}, h$ & 64.3 \\
\hline SW & $x$ & & $\mu_{2}, \mu_{3}, \mu_{8}, k_{5}, h$ & & 81.2 \\
\hline SW & & $X$ & & $\mu_{4}, \mu_{6}, \mu_{7}, \mu_{8}, h$ & 72.2 \\
\hline SW & $x$ & $X$ & $\mu_{2}, \mu_{3}, \mu_{8}$ & $k_{5}, h$ & 86.7 \\
\hline $\mathrm{BF}$ & $x$ & & $\mu_{2}, \mu_{3}, k_{4}, k_{5}, h$ & & 60.3 \\
\hline $\mathrm{BF}$ & & $x$ & & $\mu_{3}, \mu_{4}, \mu_{8}, k_{5}, h$ & 71.2 \\
\hline $\mathrm{BF}$ & $x$ & $x$ & & $\mu_{3}, \mu_{4}, \mu_{8}, k_{5}, h$ & 71.2 \\
\hline
\end{tabular}

The prediction is performed on three aneurysms sets: (1) 117 sidewall and bifurcation (SW + BF) aneurysms, (2) 58 sidewall (SW) aneurysms, and (3) 59 bifurcation (BF) aneurysms. Aneurysm model (AM) and parent vessel model (PVM) are considered both separately and combined. The set of features taken into account for each particular classification case are marked with an $\mathrm{X}$. Sequential backward selection is applied to determine best features set.

\section{Sensitivity to Segmentation}

To determine the sensitivity of the classification to segmentation, a subset of $58 \mathrm{SW}$ aneurysms are segmented using two methods, namely region-growing thresholding ${ }^{15}$ and level sets. ${ }^{19}$ For this analysis, only isolated aneurysm models are considered. The robustness analysis involves four classification cases: two in which the training and the testing sets are segmented using the same method, and two in which the training and the testing sets are segmented using different methods.

The best results are obtained when both the training and the testing sets are segmented using the same method (Table 4). In this case, both segmentation methods result in similar classification accuracy $(\approx 80 \%)$, which is reassuring. Equally encouraging is the fact that the two resulting best feature sets have four overlapping features. Segmentation does influence the result of classification when the training and the testing sets are segmented differently. It is to be expected that depending on the different natures of the various edge- and region-based segmentation algorithms, they may give somewhat different results and consequently different information. Furthermore, many segmentation methods have one or more adjustable parameters which determine the granularity of the segmentation and different tuning of these parameters may lead sometimes to significantly different results.

\section{Sensitivity to Cutting Planes Definition}

The sensitivity of the classification to cutting plane definition is investigated on four aneurysms. The aneurysms types are SW ruptured, SW unruptured, BF ruptured, and BF unruptured. All four aneurysms were correctly classified previously by writhe number analysis. Only parent vessel models are analyzed here, since they may be affected by cutting plane definition.
Several models are created by using three different cutting planes per parent vessel cut. The planes are roughly chosen at distances equal to $1.5,1$, and 0.5 of the vessel diameter as measured at the aneurysm neck. Cutting planes choices are shown in Figs. 6a and $6 \mathrm{f}$ for SW (nine models per aneurysm) and BF (27 models per aneurysm) types respectively. In the figure, the two most extreme models are shown for each aneurysm-when all adjacent vessels are cut using the outermost planes, and then all adjacent vessels are cut using the innermost planes. All other models fall between the two extreme models shown here.

The resulting aneurysm models were classified according to their type (SW or BF) using the best feature sets from Table 3. All 72 models were correctly classified regardless of the cutting plane choices (Table 5). The results suggest the classification based on writhe number analysis is not overly sensitive to cutting plane definition.

\section{Sensitivity to Scaling}

As described in "The Writhe Number" section, the writhe number of a surface depends on both the size and the shape of the surface. This dependence is analyzed on 10 unruptured SW aneurysms. The mean value of their largest diameter is $4.83 \pm 1.73 \mathrm{~mm}$. All 10 aneurysms were correctly classified as unruptured by the writhe number-based classification. The 10 aneurysms were subsequently scaled three times their size in increments of $20 \%$. Each initial aneurysm was thus associated with 10 scaled aneurysms, resulting in a total of one hundred models.

First, rupture classification was applied to the scaled aneurysms using their largest diameter as the only feature. The classifier was trained on the $58 \mathrm{SW}$ aneurysms. Each set of 10 scaled aneurysms was analyzed separately. Within each set, the aneurysms were 
3D Shape Analysis of Intracranial Aneurysms

(a)
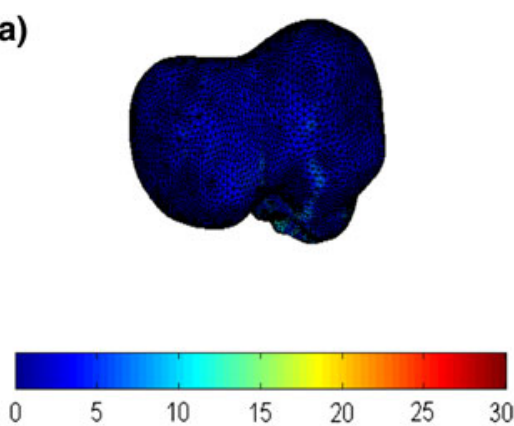

(d)

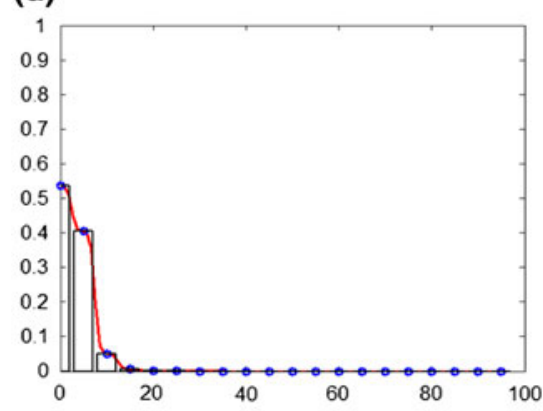

(g)
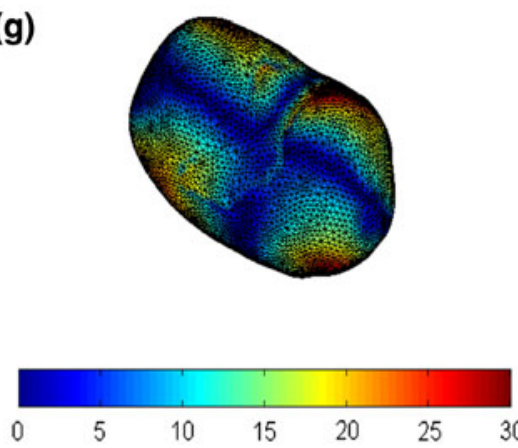

(j)

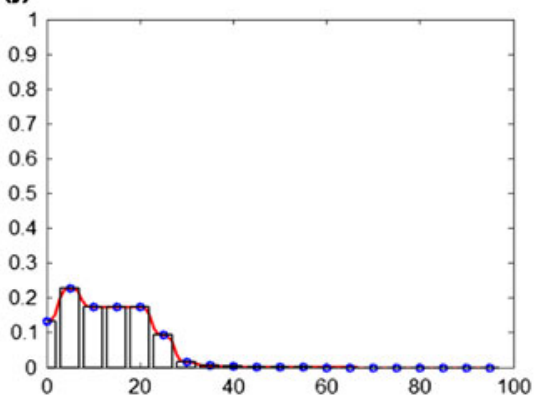

(b)
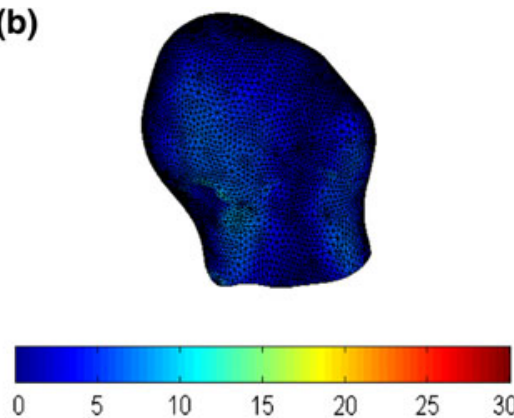

(e)

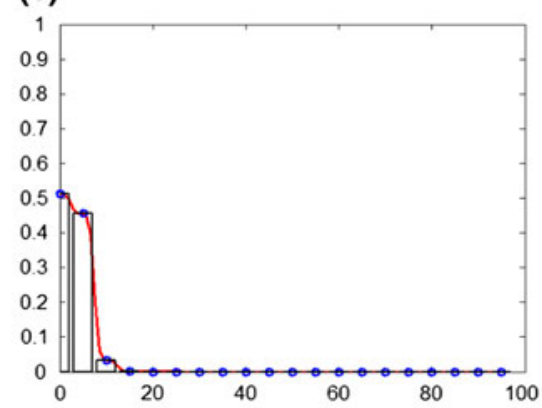

(h)
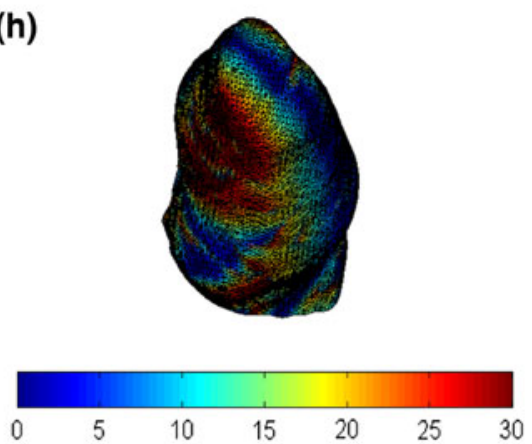

(k)

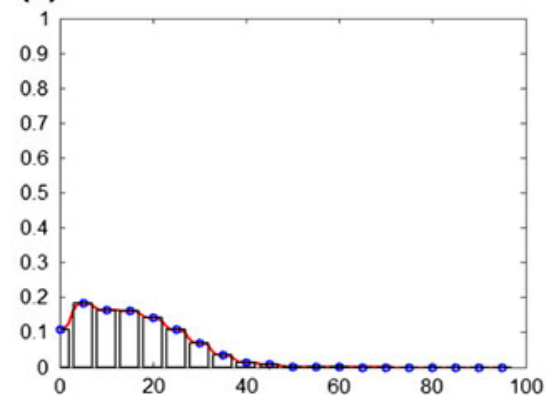

(c)
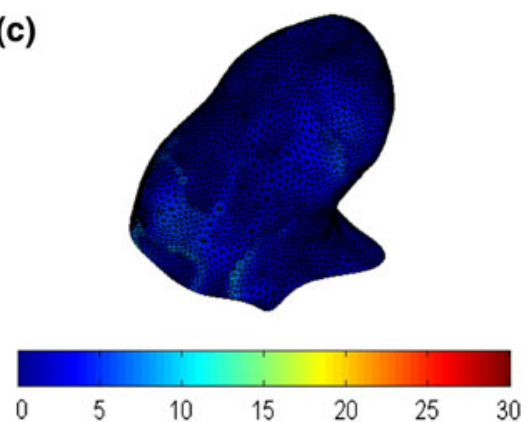

(f)

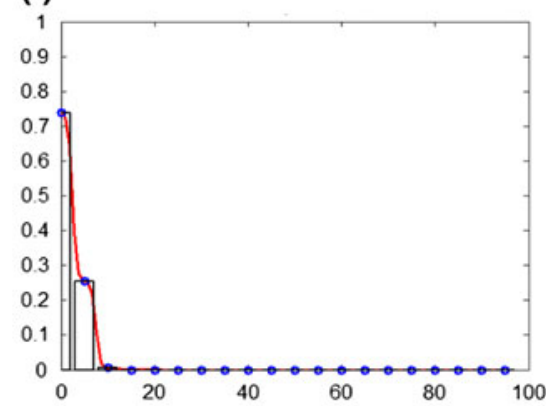

(i)
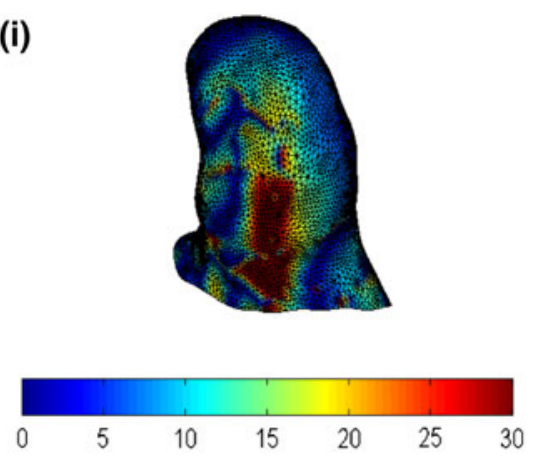

(I)

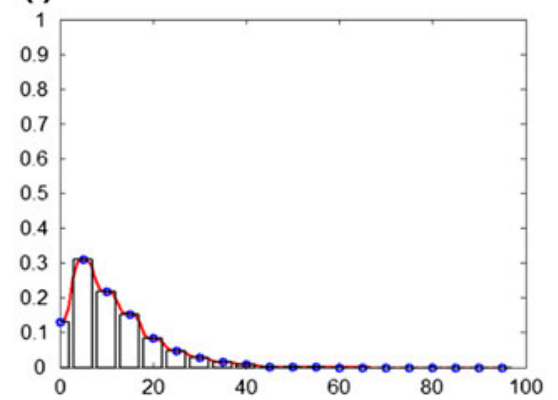

FIGURE 4. Analysis results on for representative ruptured and unruptured sidewall aneurysms. (a)-(c) Unruptured aneurysms. Writhe number values along the surface. Low values are interpreted as small twisting forces. (d)-(f) Corresponding histograms. The approximating probability distribution is shown in red. Unruptured aneurysms have sharp distributions, low entropy, and low skewness. (g)-(i) Ruptured aneurysms. Writhe number values along the surface. High values are interpreted as high twisting forces and are a predictor for rupture. (j)-(I) Corresponding histograms. The approximating probability distribution is shown in red. Ruptured aneurysms have flat distributions, high entropy, and high positive skewness.

classified in their increasing size order. The first scaled value of the largest diameter at which the classification label changed from unruptured to ruptured was recorded. For the whole set of one hundred scaled aneurysms this analysis resulted in 10 diameter values with a mean of $9.13 \pm 0.33 \mathrm{~mm}$. This indicates a tight threshold of $9 \mathrm{~mm}$ for rupture prediction based on the largest diameter. 
(a)
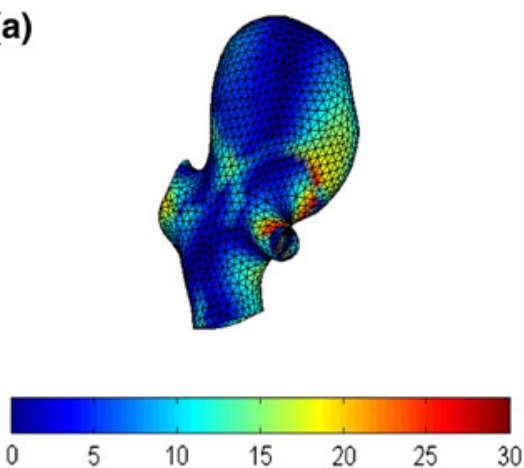

(d)

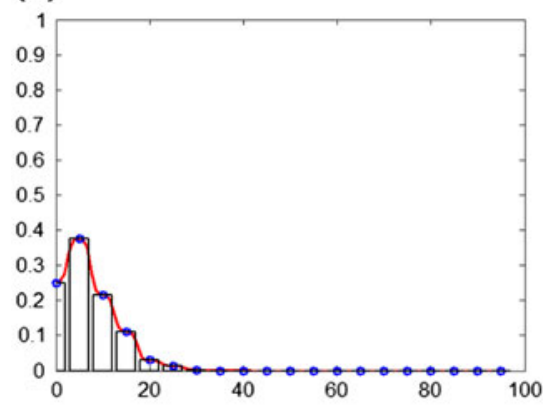

(g)
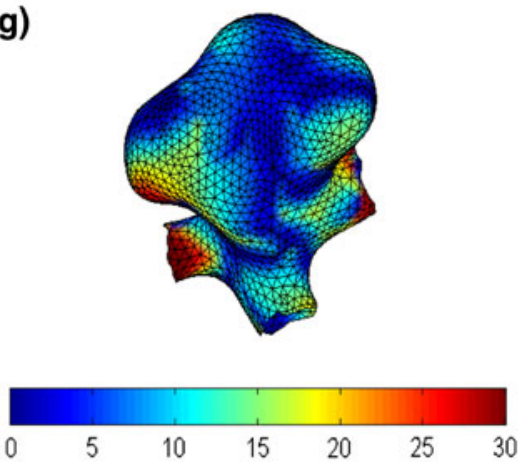

(j)

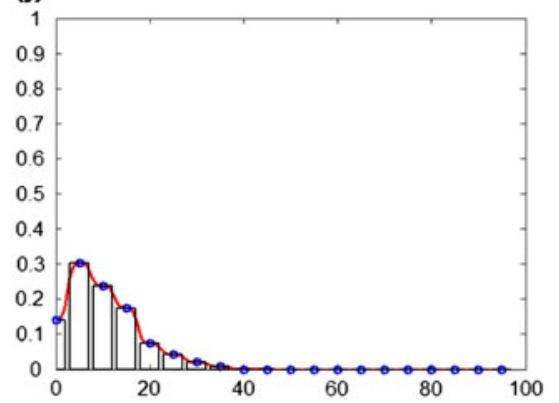

(b)
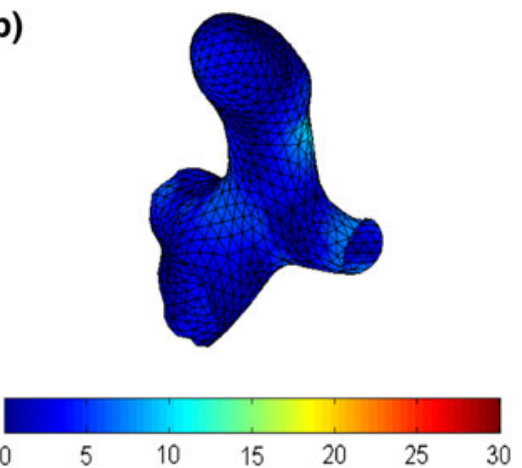

(e)

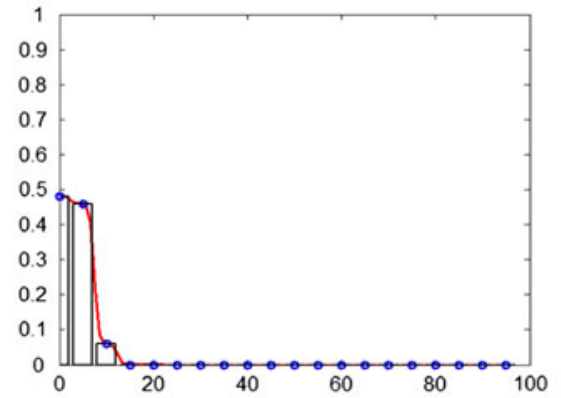

(h)
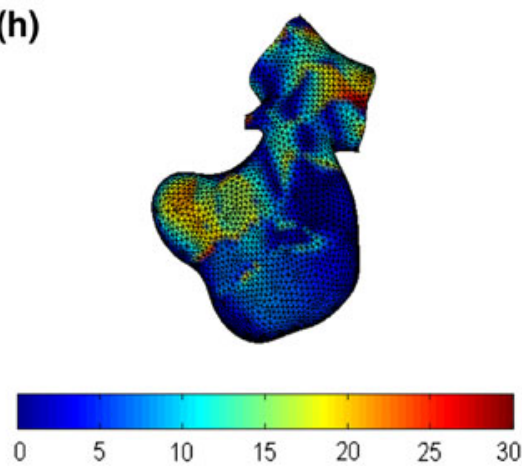

(k)

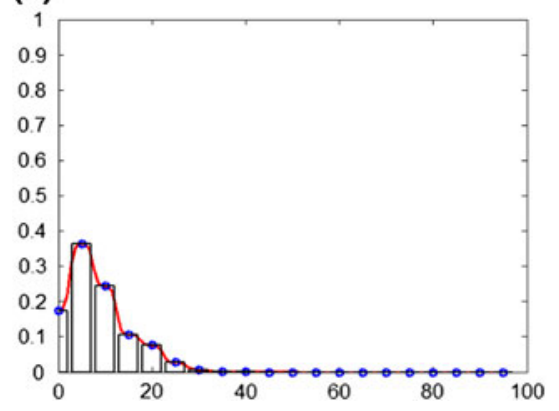

(c)
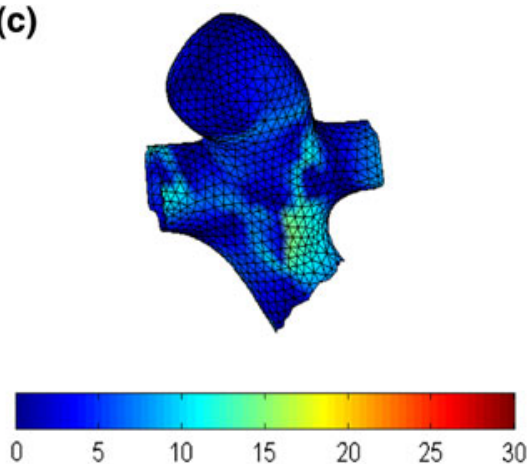

(f)

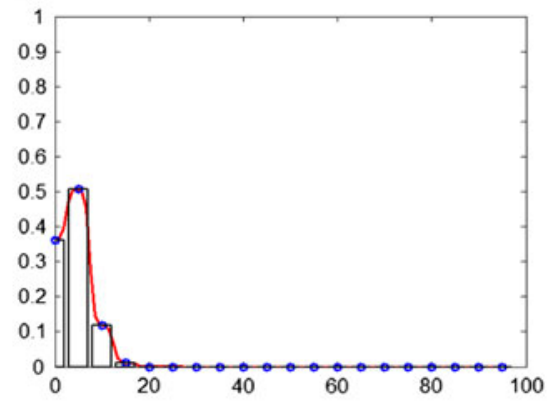

(i)
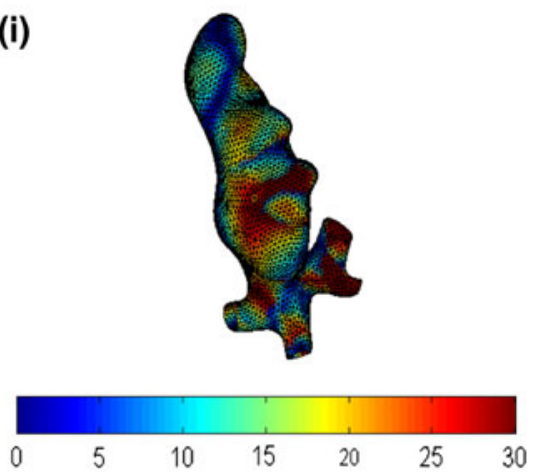

(l)

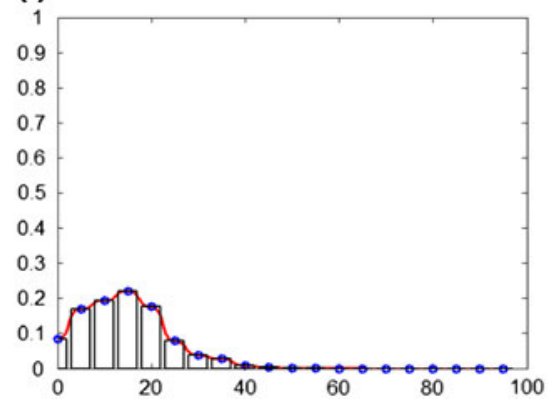

FIGURE 5. Analysis results on for representative ruptured and unruptured bifurcation aneurysms. (a)-(c) Unruptured aneurysms. Writhe number values along the surface. Low values are interpreted as small twisting forces. (d)-(f) Corresponding histograms. The approximating probability distribution is shown in red. Unruptured aneurysms have sharp distributions, low entropy, and low skewness. (g)-(i) Ruptured aneurysms. Writhe number values along the surface. High values are interpreted as high twisting forces and are a predictor for rupture. (j)-(I) Corresponding histograms. The approximating probability distribution is shown in red. Ruptured aneurysms have flat distributions, high entropy, and high positive skewness.

Second, rupture classification was applied to the scaled aneurysms using writhe number statistics, namely the best feature set corresponding to SW aneurysms from Table 3 . The classifier was trained on the $58 \mathrm{SW}$ aneurysms. Each set of 10 scaled aneurysms was analyzed separately. Within each set, the aneurysms 
TABLE 4. Sensitivity of the classification to segmentation.

\begin{tabular}{llll}
\hline Train set & Test set & Best features & Accuracy \% \\
\hline Threshold & Threshold & $\mu_{2}, \mu_{3}, \mu_{8}, k_{5}, h$ & 81.2 \\
Threshold & Level sets & $\mu_{2}, \mu_{3}, \mu_{8}, k_{5}, h$ & 76.8 \\
Level sets & Level sets & $\mu_{2}, \mu_{3}, \mu_{7}, \mu_{8}, h$ & 80.3 \\
Level sets & Threshold & $\mu_{2}, \mu_{3}, \mu_{7}, \mu_{8}, h$ & 74.6 \\
\hline
\end{tabular}

The two segmentation methods used are region-growing thresholding and level sets. The study involves four classification cases: two in which the training and the testing sets are segmented using the same method, and two in which the training and the testing sets are segmented using different methods.
TABLE 5. Sensitivity of the classification to cutting plane definition.

\begin{tabular}{llrc}
\hline Type & Ruptured? & $N$ & Correctly classified \\
\hline SW & Yes & 9 & 9 \\
SW & No & 9 & 9 \\
BF & Yes & 27 & 27 \\
BF & No & 27 & 27
\end{tabular}

Four aneurysms with parent vessels attached were cut using three different planes per parent vessel. $N$ represents the resulting number of models for each aneurysm. The models are classified according to their type (SW or BF). (a)

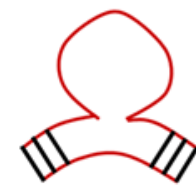

(f)

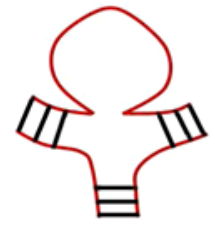

(b)

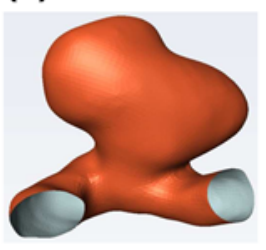

(g)

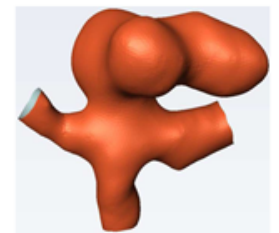

(c)

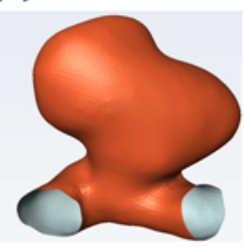

(h)

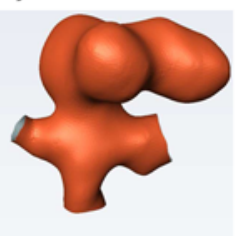

(d)

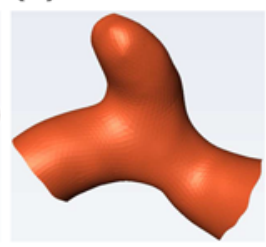

(i)

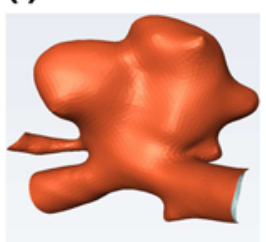

(e)

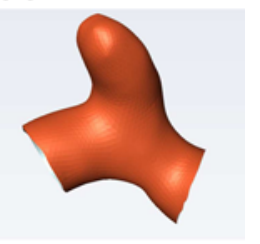

(j)

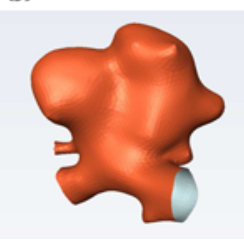

FIGURE 6. Sensitivity of the classification to cutting plane definition. Four aneurysms with parent vessels attached were cut using three different planes per parent vessel cut. (a) Sidewall aneurysm. Cutting planes options are shown in black. Combining the cutting planes result in nine models. (b) SW ruptured: outermost planes used. (c) SW ruptured: innermost planes used. (d) SW unruptured: outermost planes used. (e) SW unruptured: innermost planes used. (f) Bifurcation aneurysm. Cutting planes options are shown in black. Combining the cutting planes result in 27 models. (g) BF ruptured: outermost planes used. (h) BF ruptured: innermost planes used. (i) BF unruptured: outermost planes used. (j) BF unruptured: innermost planes used.

were classified in their increasing size order. The first scaled value of the largest diameter at which the writhe number-based classification label changed from unruptured to ruptured was recorded. For the whole set of one hundred scaled aneurysm this analysis resulted in 10 diameter values with a mean of $8.06 \pm 1.41 \mathrm{~mm}$. Both the smaller mean as well as the larger standard deviation here indicates that some aneurysms changed ruptured status at a smaller scaling factor compared to when only their largest diameter was considered. This suggests that the shape of the aneurysm as captured by the writhe number played a role in rupture status discrimination.

To determine this morphological contribution, we have analyzed the shape of the 10 initial aneurysms using the best performing shape index from Table 2, namely the NSI, which is a scale-independent index. In Fig. 7, for each of the 10 initial aneurysms we determine the smallest scale factor at which the writhe number statistics switch from unruptured to ruptured and, to capture the size of the scaled aneurysm, we compute the diameter. Figure 7, then, is a plot of NSI on the $y$ axis versus this writhe-related diameter. It is apparent from the figure that those aneurysms for which the writhe number analysis suggested rupture at a smaller scaled size, have higher NSI which suggests more complex, irregular shapes. Consequently, based on their shape variations, these aneurysms were labeled as ruptured by writhe number analysis despite their smaller scaled size.

\section{DISCUSSION}

SAH as a consequence of aneurysm rupture is a lifethreatening and debilitating event with high mortality and morbidity rates. While current clinical practice relies mostly on size of the aneurysms as the main indicator for rupture, this study suggests that morphology is likely to play an important role in discriminating between ruptured and unruptured cerebral 


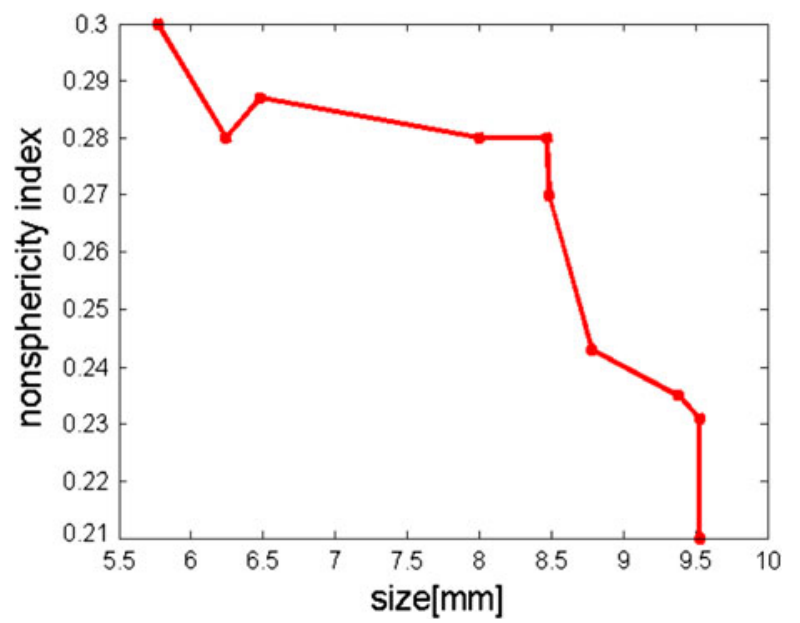

FIGURE 7. Writhe number analysis of scaled aneurysms. For each of the 10 initial aneurysm the non-sphericity index is plotted vs. the smallest scaled value of the largest diameter at which writhe number analysis predicted rupture. It is apparent from the figure, that same smaller scaled aneurysm were labeled as ruptured by the writhe number analysis, based on their irregular shape as suggested by a large non-sphericity index.

aneurysms. $^{4,13,16}$ The morphological analysis in this work is based on writhe number distributions on the surface of aneurysms. To the best of our knowledge, this is the first study using 3D shape descriptors derived from the writhe number statistics. The results show that ruptured aneurysms tend to have regions of high writhe number values, which can be interpreted as high twisting forces acting on the surface and are associated with shape asymmetries. These qualities are captured by the writhe number and play a role in rupture status discriminations.

This study is performed both on isolated aneurysmal dome models and on combined models incorporating the attached adjacent parent vessel region. When only the aneurysmal dome is considered the classification yields encouraging results on the SW aneurysm subset, but performs poorly on BF aneurysm subset ( 81.2 vs. $60.3 \%$ accuracy). Incorporating parent vessel information into the classification process results in an increase in accuracy for BF subgroup $(60.3-71.2 \%)$. The fact that the best feature set for BF aneurysms contains only parent vessel features suggests that the relation between the aneurysm and the adjacent vessels is a better indicator of rupture compared to the shape of the aneurysm sac in the BF subset. Still, this morphological analysis captures the rupture risk of SW aneurysms much better than that of BF aneurysms ( 86.7 vs. $71.2 \%$ accuracy). There seems to be a morphological split between SW and BF aneurysms which is supported by recent research in our lab using two-dimensional shape descriptors such as the AR index, HW ratio, and inflow-angle into the aneurysm dome. The fact that all indexes studied here performed poorly on $\mathrm{BF}$ aneurysms suggests that rupture status discrimination is more challenging in $\mathrm{BF}$ aneurysms compared to SW aneurysms. More studies are needed to confirm and gain a better understanding of this phenomenon.

Robustness analysis shows the classification to be insensitive to segmentation as long as the same method is used on both the training and the testing sets. The classification is sensitive to segmentation when the training and testing sets are segmented using different methods. Segmentation of cerebral vasculature is a challenging task and the research in the field remains active. It is beyond the scope of this article to improve segmentation algorithms or to compare the performance of the various methods. At this moment, our recommendation is to use compatible training and testing methods for rupture classification.

When creating AMs with parent vessels attached, the cutting planes are chosen somewhat arbitrarily. While there is some research on creating AMs, ${ }^{5}$ we do not know of the existence of a comprehensive study to recommend a methodology on how to create parent vessel models. Our analysis suggests the classification based on writhe number is relatively impervious to cutting plane definitions. However, since incorporating parent vessel information improves the prediction results, it would be worth exploring the effect of cutting plane definition on a larger set of both correctly classified and misclassified aneurysms.

Similar to previous morphological research, the current study premise is based on an yet unproven assumption that aneurysms do not change shape and size upon rupture. ${ }^{12,13,16,20}$ This is still a point of contention and there is not enough information to decidedly settle the debate. Some studies report no major change in size and shape after rupture, ${ }^{20}$ whereas the ISUIA study ${ }^{22}$ contends that ruptured aneurysms data should not be used to draw conclusions about unruptured aneurysms evolution. If indeed the aneurysm changes size and shape following rupture, it remains to be determined if shape and surface indexes, such as the writhe number, are more sensitive in capturing these post-rupture changes than size indexes and thus, less relevant to the rupture prediction process.

The current analysis, similar to recent morphological research, is performed retrospectively on a data set of aneurysms which were labeled as being ruptured or not at the time of presentation by the treating neurosurgeons. The temporal aneurysm shape evolution and clinical symptomatology were not followed clinically over a period of time, and were not available prior to rupture. This limitation has been previously discussed and addressed by Dhar et al. ${ }^{4}$ In order to serve as a clinical predictor, the results will require validation in a prospective clinical study. 
The purpose of this work is to introduce the writhe number for shape analysis and as such the rupture status classification is done using exclusively writhe number derived features. It is very likely that combining morphological features like those presented here and size features like aneurysm dimensions, including height, width, and neck diameter, will increase the discrimination accuracy and will result in a more complete understanding of intracranial aneurysms and their natural progression to rupture. We intend to incorporate both size and shape features in a future comprehensive study.

While the analysis was performed on a relatively large database and the results are very encouraging, the eventual added value of the method remains to be determined in the clinical setting and, as mentioned above, would require validating in prospective clinical trials.

In summary, the writhe number of surfaces is introduced as a method to analyze the complex shape of intracranial aneurysms and classify their rupture status. A morphological dichotomy is reported between SW and $\mathrm{BF}$ aneurysms, and the two subtypes are analyzed separately. The analysis is performed both with and without adjacent parent vessels information and leads us to conclude that the relation between the aneurysmal sac and surrounding vessels is relevant for rupture status prediction. The classification accuracy obtained using morphological analysis based on writhe number was of $86 \pm 2 \%$ for SW aneurysms and of $71 \pm 3 \%$ for $\mathrm{BF}$ aneurysms.

\section{ELECTRONIC SUPPLEMENTARY MATERIAL}

The online version of this article (doi:10.1007/ s10439-010-0241-x) contains supplementary material, which is available to authorized users.

\section{REFERENCES}

${ }^{1}$ Beer, F. P., E. R. Johnston Jr., E. R. Eisenberg, and G. H. Staab. Vector Mechanics for Engineers: Statics. Ohio: McGraw-Hill Science, 2003.

${ }^{2}$ Bowman, A. W. and A. Azzalini. Applied Smoothing Techniques for Data Analysis. New York: Oxford University Press, 1997.

${ }^{3}$ Coert, B. A., S. D. Chang, H. M. Do, M. P. Marks, and G. K. Steinberg. Surgical and endovascular management of symptomatic posterior circulation fusiform aneurysms. J. Neurosurg. 106:855-865, 2007.

${ }^{4}$ Dhar, S., M. Tremmel, J. Mocco, M. Kim, J. Yamamoto, A. H. Siddiqui, L. N. Hopkins, and H. Meng. Morphology parameters for intracranial aneurysm rupture risk assessment. Neurosurgery 63(2):185-197, 2008.

${ }^{5}$ Ford, M. D., Y. Hoi, M. Piccinelli, L. Antiga, and D. A. Steinman. An objective approach to digital removal of saccular aneurysms: technique and applications. $\mathrm{Br} . \mathrm{J}$. Radiol. 82:55-61, 2009.

${ }^{6}$ Fuller, F. B. The writhing number of a space curve. Proc. Natl. Acad. Sci. USA 68(4):815-819, 1971.

${ }^{7}$ Hardle, W. Applied Nonparametric Regression. Cambridge: Cambridge University Press, 1990.

${ }^{8}$ Harrell, F. E. Regression Modeling Strategies. Springer Series in Statistics, 2001.

${ }^{9}$ Hoh, B. L., C. L. Sistrom, C. S. Firment, G. L. Fautheree, G. J. Velat, J. H. Whiting, J. F. Reavey-Cantwell, and S. B. Lewis. Bottleneck factor and height-width ratio: association with ruptured aneurysms in patients with multiple cerebral aneurysms. Neurosurgery 61(4):716-723, 2007.

${ }^{10}$ Hurdal, M. K., J. B. Gutierrez, C. Laing, and D. A. Smith. Shape analysis for automated sulcal classification and parcellation of MRI data. J. Comb. Optim. 15(3):257-275, 2008.

${ }^{11}$ Lauric, A., E. Miller, S. Frisken, and A. M. Malek. Automated detection of intracranial aneurysms based on parent vessel 3D analysis. Med. Imaging Anal. 14:149-159, 2010.

${ }^{12}$ Ma, B. S., R. E. Harbaugh, and M. L. Raghavan. Threedimensional geometrical characterization of cerebral aneurysms. Ann. Biomed. Eng. 32(2)264-273, 2004.

${ }^{13}$ Millan, R. D., L. Dempere-Marco, J. M. Pozo, J. R. Cebral, and A. F. Frangi. Morphological characterization of intracranial aneurysms using 3-d moment invariants. IEEE Trans. Med. Imaging 26(9):1270-1282, 2007.

${ }^{14}$ Millington, I. Game Physics Engine Development. Menlo Park: Morgan Kaufmann, 2007.

${ }^{15}$ Pham, D. L., C. Xu, and J. L. Prince. Current methods in medical image segmentation. Annu. Rev. Biomed. Eng. 2:315-337, 2000.

${ }^{16}$ Raghavan, M. L., B. Ma, and R. E. Harbaugh. Quantified aneurysm shape and rupture risk. J. Neurosurg. 102(2):355$362,2005$.

${ }^{17}$ Rohde, S., K. Lahmann, J. Beck, R. Nafe, B. Yan, A. Raabe, and J. Berkefeld. Fourier analysis of intracranial aneurysms: towards an objective and quantitative evaluation of the shape of aneurysms. Neuroradiology 47(2):121$126,2005$.

${ }^{18}$ Rossetto, V. and A. C. Maggs. Writhing geometry of open DNA. J. Chem. Phys.118:9864-9874, 2003.

${ }^{19}$ Sethian, J. A. Level Set Methods and Fast Marching Methods. Cambridge: Cambridge University Press, 1999.

${ }^{20}$ Ujiie, H., H. Tachibana, O. Hiramatsu, A. L. Hazel, T. Matsumoto, Y. Ogasawara, H. Nakajima, T. Hori, K. Takakura, and F. Kajiya. Effects of size and shape (aspect ratio) on the hemodynamics of saccular aneurysms: a possible index for surgical treatment of intracranial aneurysms. Neurosurgery 45(1):119-130, 1999.

${ }^{21}$ Wardlaw, J. M. and P. M. White. The detection and management of unruptured intracranial aneurysms. Brain 123(2):205-221, 2000.

${ }^{22}$ Wiebers, D. O. Unruptured intracranial aneurysms: natural history, clinical outcome, and risks of surgical and endovascular treatment. Lancet 362(9378):103-110, 2003.

${ }^{23}$ Wiebers, D. O. Patients with small, asymptomatic, unruptured intracranial aneurysms and no history of subarachnoid hemorrhage should generally be treated conservatively: for. Stroke 36:408-409, 2005.

${ }^{24}$ Wolfe, S. Q., M. K. Baskaya, R. C. Heros, and R. P. Tummala. Cerebral aneurysms: learning from the past and looking toward the future. Clin. Neurosurg. 53:157-178, 2006. 